\title{
Scanning anisotropy parameters in complex media
}

\author{
Tariq Alkhalifah ${ }^{1}$
}

\begin{abstract}
Parameter estimation in an inhomogeneous anisotropic medium offers many challenges; chief among them is the trade-off between inhomogeneity and anisotropy. It is especially hard to estimate the anisotropy anellipticity parameter $\eta$ in complex media. Using perturbation theory and Taylor's series, I have expanded the solutions of the anisotropic eikonal equation for transversely isotropic (TI) media with a vertical symmetry axis (VTI) in terms of the independent parameter $\eta$ from a generally inhomogeneous elliptically anisotropic medium background. This new VTI traveltime solution is based on a set of precomputed perturbations extracted from solving linear partial differential equations. The traveltimes obtained from these equations
\end{abstract}

serve as the coefficients of a Taylor-type expansion of the total traveltime in terms of $\eta$. Shanks transform is used to predict the transient behavior of the expansion and improve its accuracy using fewer terms. A homogeneous medium simplification of the expansion provides classical nonhyperbolic moveout descriptions of the traveltime that are more accurate than other recently derived approximations. In addition, this formulation provides a tool to scan for anisotropic parameters in a generally inhomogeneous medium background. A Marmousi test demonstrates the accuracy of this approximation. For a tilted axis of symmetry, the equations are still applicable with a slightly more complicated framework because the vertical velocity and $\delta$ are not readily available from the data.

\section{INTRODUCTION}

The ability to scan efficiently and effectively for anisotropy parameters in a generally inhomogeneous medium is essential to build credible anisotropic models. Audebert et al. (1997) develops a common-reflection-point scan for velocity in the isotropic case; they show that, through perturbation theory, the traveltime is linearly scaled by velocity perturbations. For anisotropic media, the relation between media parameters and the traveltime is far more complicated because the ray direction is different from the phase direction, and thus the traveltime integral is highly nonlinear with respect to the anisotropic parameters.

Traveltimes are conventionally evaluated by solving the zeroorder (high-frequency) approximation of the Wentzel, Kramers, and Brillouin (WKB) expansion of the wave equation. This high-frequency approximation solution is sufficient for most practical imaging applications. It provides us with a traveltime equation better known as the eikonal equation. The eikonal equation is a nonlinear partial differential equation that can be solved using any of the familiar numerical methods. Among the most popular of these methods are the ray tracing and finite-difference approaches. Finite-difference solutions of the eikonal equation have been recognized as one of the most efficient means of traveltime calculations (Vidale, 1990; van Trier and Symes, 1991; Popovici, 1991; Alkhalifah and Fomel, 2001). Some main advantages of this method in comparison to ray tracing include the ability to directly provide solutions on regular grids, a complete coverage of the solution space, and a high numerical robustness. However, the method only provides traveltimes corresponding to the first arrival, which might be not the most energetic arrival.

In anisotropic media, traveltime computation is dependent on more than one parameter to describe the velocity field. Through careful parameterization of transversely isotropic (TI) media with a vertical symmetry axis (VTI), P-wave traveltimes become, for practical purposes, dependent on only three parameters to describe the velocity field. Alkhalifah and Tsvankin (1995) further demonstrate that a new representation in terms of just two parameters is sufficient for performing all time-related processing, such as NMO correction (including nonhyperbolic

Manuscript received by the Editor 11 May 2010; revised manuscript received 30 September 2010; published online 21 March 2011.

${ }^{1}$ King Abdullah University of Science and Technology (KAUST), Physical Sciences and Engineering (PSE) Division, Saudi Arabia. E-mail: tariq. alkhalifah@kaust.edu.sa.

(C) 2011 Society of Exploration Geophysicists. All rights reserved. 
moveout correction, if necessary), dip-moveout removal, and prestack and poststack time migration. These two parameters are the NMO velocity $v$ and an anisotropy parameter referred to as $\eta$ (or the horizontal velocity $v_{h}$ ) that relates the NMO velocity to the horizontal one. As a result, the third parameter, the vertical velocity $v_{v}$, controls the depth placement of reflections. This is evident in the eikonal equation for VTI media developed by Alkhalifah (1998, 2000).

The process of finding a stable solution for this eikonal equation using finite-difference schemes generally is hard, especially because such a process requires finding the root of a quartic equation at each computational step. However, the traveltime computation for a slightly more simplified, but not practical, anisotropic model, referred to as elliptical anisotropy, is far more efficient. The reason for the high efficiency is that elliptical anisotropy has the same order of complexity (nonlinearity) in the eikonal equation as the isotropic equation has. Thus, although elliptical anisotropy represents an uncommon model in practice, it provides some flexibility in treating the difference between vertical and horizontal velocities, or in other terms, the flexibility of stretching the depth axis to obtain accurate reflection depths in imaging (Peng and Steenson, 2001). However, elliptical anisotropy does not provide accurate focusing for media of VTI anisotropy.

The nonlinear nature of the eikonal partial differential equation was first challenged by Aldridge (1994), who linearized the eikonal equation with respect to velocity perturbation while retaining its first-order nature, and showed its direct relation to the familiar traveltime perturbation equation used in tomography. Ettrich and Gajewski (1998) used perturbation theory to compute traveltimes in anisotropic media, perturbing with a background velocity model that is elliptically anisotropic. Meanwhile, Alkhalifah (2002) developed an eikonal-based linearization formula for perturbations in anisotropic parameters and solved it numerically using the fast marching method (Sethian, 1996). The linear feature increased the efficiency and stability of the numerical solution, especially in the anisotropic case. To compute traveltimes for anisotropic media using the linearized eikonal equation, we also perturb from a background elliptically anisotropic inhomogeneous model, especially because, for such a model, we can compute traveltimes efficiently.

Prestack depth migration has been used heavily to help estimate the velocity field (Al-Yahya, 1989). Migration velocity analysis requires repeated applications of migration for various velocity models, and as a result, repeated traveltime calculation using various velocity models obtained during the velocity analysis process. Among the update schemes for such a process is traveltime tomographic inversion. A linearized eikonal equation with respect to anisotropic parameters can help toward improving such an inversion. In particular, having the ability to do efficient scans over desired media parameters can help the inversion process for anisotropic parameters. Thus, linearized formulation of the VTI eikonal equation not only improves the efficiency of traveltime calculation, it also provides a platform for parameter estimation.

In this paper, I derive a high-order expansion of the traveltimes as a function of $\eta$ with coefficients estimated using linearized forms of the eikonal equation. The accuracy of such an expansion is further enhanced using the Shanks transform (an approach to predict the behavior of a series from the first few terms) to obtain better high-order representation. A scheme for scanning over $\eta$ is suggested and later tested. I also suggest a framework to extend this approach to media with a tilted axis of symmetry (TTI).

\section{THE VTI EIKONAL AND EXPANSION IN $\eta$}

The eikonal equation, describing the traveltime propagation in an isotropic medium, has the following form:

$$
v^{2}(x, y, z)(\nabla \tau)^{2}=1
$$

where $\tau(x, y, z)$ is the traveltime (eikonal) measured from the source to the point with the coordinates $(x, y, z)$, and $v$ is the velocity at that point. I will use $v$ throughout the paper to represent the NMO velocity, which is equivalent to the medium velocity for isotropic media. To formulate a well-posed initialvalue problem on equation 1 , it is sufficient to specify $\tau$ at some closed surface and to choose one of the two solutions, the wave going from or to the source.

For elliptically anisotropic media (Dellinger and Muir, 1988), the eikonal equation has the form

$$
v^{2}(x, y, z)\left(\left(\frac{\partial \tau}{\partial x}\right)^{2}+\left(\frac{\partial \tau}{\partial y}\right)^{2}\right)+v_{v}^{2}(x, y, z)\left(\frac{\partial \tau}{\partial z}\right)^{2}=1,
$$

where $v=v_{h}$, where $v_{h}$ is the horizontal velocity. This equation is very similar to equation 1 except for different coefficients in front of the vertical and horizontal traveltime derivatives, given by the vertical and horizontal velocities, respectively. The different coefficients result in an elliptical wavefront in homogeneous media, as well as an elliptical traveltime relation at each step of the eikonal equation finite-difference computation.

For VTI media, the eikonal equation (Alkhalifah, 2000) for an acoustic medium approximation differs considerably from that for isotropic or elliptically anisotropic media and has the form

$$
\begin{aligned}
& v^{2}(1+2 \eta)\left(\left(\frac{\partial \tau}{\partial x}\right)^{2}+\left(\frac{\partial \tau}{\partial y}\right)^{2}\right) \\
& +v_{v}^{2}\left(\frac{\partial \tau}{\partial z}\right)^{2}\left(1-2 \eta v^{2}\left(\left(\frac{\partial \tau}{\partial x}\right)^{2}+\left(\frac{\partial \tau}{\partial y}\right)^{2}\right)\right)=1 .
\end{aligned}
$$

The level of nonlinearity in this quartic (in terms of $\tau$ ) equation is higher than that for the isotropic or elliptically anisotropic eikonal equations. This results in much more complicated finite-difference solution approximations of the VTI eikonal equation. The nonlinearity in the eikonal equation is essential for producing multiple branches of the solution. Multivalued eikonal solutions can include different types of waves (direct, reflected, diffracted, head, and so on) as well as different branches of caustics.

Equation 3 can be solved numerically by solving a quartic equation (instead of the quadratic in the isotropic and elliptical anisotropic case), or can be solved approximately by solving a series of simpler linear equations using perturbation theory (Bender and Orszag, 1978). By considering $\eta$ constant and small, we can represent the traveltime solution as a series expansion in $\eta$. This will result in a solution that is globally representative in the space domain, and despite the approximation of small $\eta$, the 
accuracy, as we will see later, for even large $\eta$ is good. The constant $\eta$ assumption assumes a factorized medium in $\eta$ (useful for an $\eta$ model-development application). However, all other velocities, including $v_{v}$ and $v$ (or $\delta$ ), are allowed to vary freely.

Figure 1 illustrates the concept of the global expansion as we predict the traveltime for any $\eta$ from its behavior around $\eta=0$ for the full traveltime field. Specifically, we substitute the following trial solution,

$$
\begin{aligned}
\tau(x, y, z) \approx & \tau_{0}(x, y, z)+\tau_{1}(x, y, z) \eta+\tau_{2}(x, y, z) \eta^{2} \\
& +\tau_{3}(x, y, z) \eta^{3}
\end{aligned}
$$

where $\tau_{0}, \tau_{1}, \tau_{2}$, and $\tau_{3}$ are coefficients of the expansion with units of traveltime, into the eikonal equation 3. For practical purposes, I consider here only four terms of the expansion. As a result, and as shown in Appendix $\mathrm{A}, \tau_{0}$ satisfies the eikonal equation for elliptical anisotropy, which is easily solvable, whereas $\tau_{1}$, $\tau_{2}$, and $\tau_{3}$ satisfy a linear first-order partial differential equation having, as shown in Appendix A, the following general form:

$$
v_{v}^{2} \frac{\partial \tau_{0}}{\partial z} \frac{\partial \tau_{i}}{\partial z}+v^{2} \frac{\partial \tau_{0}}{\partial y} \frac{\partial \tau_{i}}{\partial y}+v^{2} \frac{\partial \tau_{0}}{\partial x} \frac{\partial \tau_{i}}{\partial x}=f_{i}(x, y, z),
$$

with $i=1,2,3$. The function $f_{i}(x, y, z)$ is the source term shown in Appendix A, and this function gets more complicated as $i$ is larger and it depends on terms that can be evaluated only in sequence. Therefore, these linear partial differential equations must be solved in succession starting with $i=1$.

As soon as the $\tau_{1}$, and $\tau_{2}$ are evaluated using the fast marching method as described in detail later, they can be used to predict the traveltime using the first sequence of the Shanks transform (Bender and Orszag, 1978), which, as shown in Appendix A, has the form

$$
\tau(x, y, z) \approx \tau_{0}(x, y, z)+\frac{\eta \tau_{1}^{2}(x, y, z)}{\tau_{1}(x, y, z)-\eta \tau_{2}(x, y, z)} .
$$

To obtain higher order accuracy, we solve an additional linear partial differential equation for $\tau_{3}$, and use the second sequence of the Shanks transform (Bender and Orszag, 1978) to obtain the following traveltime representation:

$$
\begin{aligned}
\tau(x, y, z) \approx & \tau_{0}(x, y, z) \\
& +\eta\left(\tau_{1}(x, y, z)+\frac{\eta \tau_{2}^{2}(x, y, z)}{\tau_{2}(x, y, z)-\eta \tau_{3}(x, y, z)}\right) .
\end{aligned}
$$

Choosing between equations 6 and 7 depends on the importance of accuracy versus computational efficiency. For $\eta$ scan applications, the coefficients $\left(\tau_{0}, \tau_{1}, \tau_{2}\right.$, and $\left.\tau_{3}\right)$ need to be evaluated only once and can be used with either equations 6 or 7 to search for the best traveltime fit.

\section{A HOMOGENEOUS-MODEL TEST}

Although the equations above are developed for a generally inhomogeneous medium, I first examine their accuracy in representing VTI traveltime and traveltime moveout in the homogeneous case. This is natural, considering that most parameter scans are applied for an effective homogeneous-medium framework.

For simplicity, and with no loss in generality because elliptical anisotropy is of the same order of complexity as the iso- tropic case, I consider a 2D medium with $v=v_{v}$, which implies an isotropic background medium. As shown in Appendix B, I use the simple traveltime relation for the isotropic-homogeneous background assumption to solve recursively for the coefficients of the traveltime expansion in $\eta$. Thus, I obtain analytic representation for traveltimes $\tau_{0}, \tau_{1}$, and $\tau_{2}$ necessary to evaluate the first sequence of the Shanks transform using equation 6, which, as a result, is given by

$$
\tau(x, z)=\frac{\sqrt{x^{2}+z^{2}}\left((\eta+2) x^{4}+4(3 \eta+1) x^{2} z^{2}+2 z^{4}\right)}{v\left((3 \eta+2) x^{4}+4(3 \eta+1) x^{2} z^{2}+2 z^{4}\right)} .
$$

For higher order accuracy, and a more complicated representation, the second sequence of the Shanks transform approximation, as shown in Appendix B, is given by

$$
\begin{aligned}
\tau(x, z)= & \frac{(6-(\eta-4) \eta) x^{10}+8(\eta(2 \eta+5)+6) x^{8} z^{2}+4((69-16 \eta) \eta+33) x^{6} z^{4}}{2 v\left(x^{2}+z^{2}\right)^{3 / 2}\left((5 \eta+3) x^{6}+2(14 \eta+9) x^{4} z^{2}+(104 \eta+27) x^{2} z^{4}+12 z^{6}\right)} \\
& +\frac{56(8 \eta+3) x^{4} z^{6}+2(104 \eta+51) x^{2} z^{8}+24 z^{10}}{2 v\left(x^{2}+z^{2}\right)^{3 / 2}\left((5 \eta+3) x^{6}+2(14 \eta+9) x^{4} z^{2}+(104 \eta+27) x^{2} z^{4}+12 z^{6}\right) .}
\end{aligned}
$$

These formulas basically represent moveout equations for traveltime in VTI media as a function of offset and can be compared with well-known equations out there. Hake et al. (1984) derive a three-term Taylor series expansion for the moveout of reflections

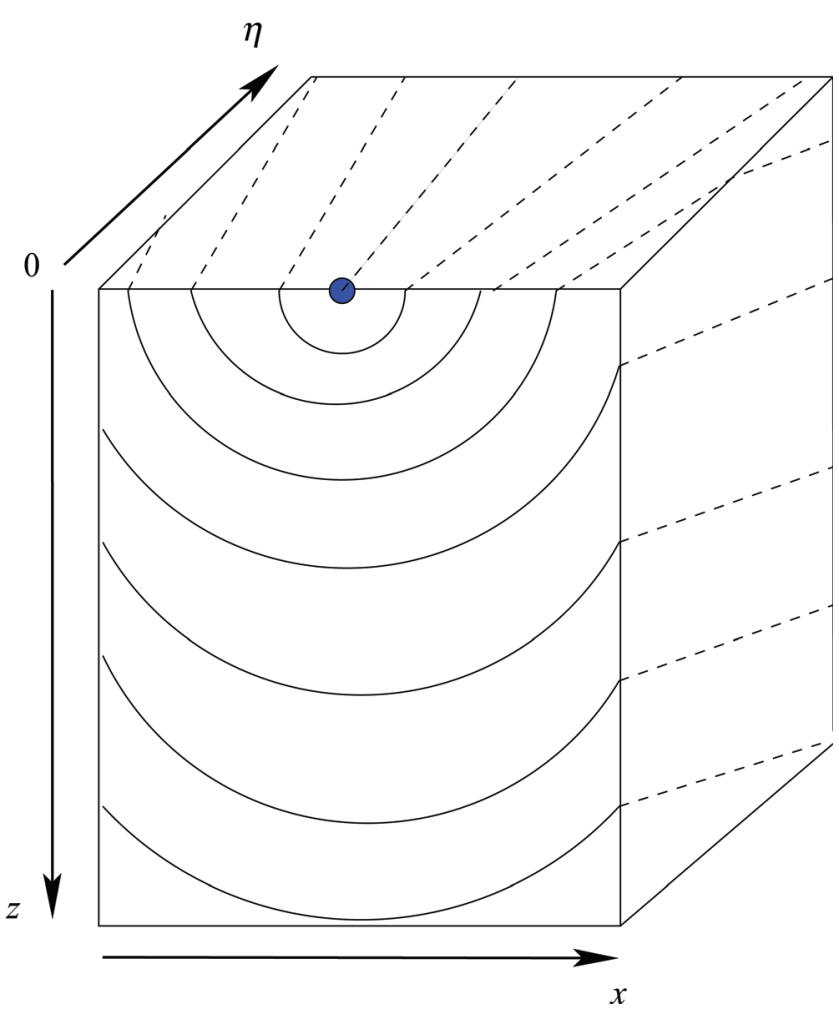

Figure 1. A schematic plot showing the relation between a background traveltime field for $\eta=0$ and when $\eta$ is larger than zero. The round dot at the top of the $\eta=0$ plane represents a source. As $\eta$ increases, the traveltime, especially in the horizontal direction, will increase depending on the approximation used in the Taylor series expansion (i.e., linearly for a simple first-order expansion). 
from horizontal interfaces in homogeneous VTI media. Such an equation with a correction factor to the nonhyperbolic term that increases the accuracy and stabilizes traveltime moveout at far offsets, when expressed in terms of $\eta$ and depth $z$, and slightly manipulated (Alkhalifah and Tsvankin, 1995; Alkhalifah, 1997), is given by

$$
t^{2}(x)=\frac{x^{2}+z^{2}}{v^{2}}-\frac{2 \eta x^{4}}{v^{2}\left[z^{2}+(1+2 \eta) x^{2}\right]} .
$$

Fomel (2004) presents a highly accurate moveout equation based on the shifted-hyperbola concept, which, written in terms of $\eta$ and depth, has the following form:

$$
t^{2}(x)=\frac{x^{2}+z^{2}}{v^{2}}-\frac{4 \eta x^{4}}{\frac{(8 \eta(\eta+1)+1) x^{2}}{v^{2}(2 \eta+1)}+\frac{1}{v^{2}} \sqrt{\frac{x^{4}}{(2 \eta+1)^{2}}+\frac{2(8 \eta(\eta+1)+1) x^{2} z^{2}}{2 \eta+1}+z^{4}}+\frac{z^{2}}{v^{2}}} .
$$

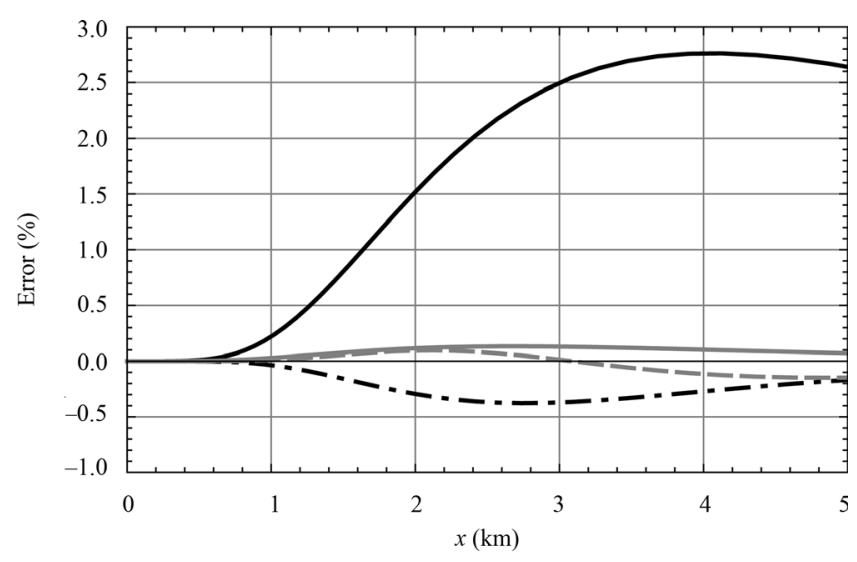

Figure 2. The traveltime error as a function of offset for a model with $v=2 \mathrm{~km} / \mathrm{s}, \eta=0.4$, and a reflector depth of $z=2 \mathrm{~km}$ for the modified Hake representation (solid black curve), the shiftedhyperbola-based one (solid gray curve), the first sequence of the Shanks transform (dashed black curve), and the second sequence of the Shanks transform (dashed gray curve). Because the depth of the reflector is $2 \mathrm{~km}$, the offset-to-depth ratio extends to 2.5 .
This equation has an additional square root in the denominator when compared to the equations shown above. This additional square root is instrumental in boosting the accuracy of the equation, but it also results in slower evaluations computationally.

Considering a model with a $2-\mathrm{km} / \mathrm{s}$ velocity and $\eta=0.4$, we compare the moveout equations extracted from our eikonalbased formulations with those used for pure moveout representation, equations 10 and 11 . For a reflector at depth $z=2 \mathrm{~km}$, Figure 2 shows the percentage traveltime errors as a function of offset for the equations given above. Clearly, the modified Hake representation (solid black curve), equation 10, is less accurate in describing the traveltime behavior than the shifted-hyperbola formula (solid gray curve), equation 11. However, the newly extracted relations corresponding to equations 8 and 9 (dashed curves) provide the level of accuracy achieved by Fomel (2004) with fewer square-root evaluations, with the second Shanks transform sequence traveltime (dashed gray curve) having an exceptional fit to the exact traveltime.

In fact, for a more practical case in which $\eta=0.2$, the errors, as shown in Figure 3a, are again comparable for the three superior formulas; but a closer look at Figure $3 \mathrm{~b}$ shows that the two newly derived Shanks-based representations provide a better fit near zero offset, with the higher sequence doing better, as expected. Consider that these equations and any higher sequence representation of the Shanks transform includes only one evaluation of a square root, as opposed to two in the case of the shifted hyperbola, which provides a clear advantage for the new equations that helps the computational speed.

To examine the error over a range of $\eta$ values (synonymous to a scan over $\eta$ ), Figure 4 shows a comparison of the traveltime errors for the second sequence of the Shanks transform (Figure 4a) with the shifted-hyperbola-based formula (Figure 4b) for a range of offsets and $\eta$ values. Again, despite the lower errors of the shifted hyperbola for large $\eta$ at large offsets, the Shanks transform formula provides better accuracy at lower offsets, given by a larger near-zero-error region.

Finally, we examine the residual traveltime shift between an isotropic model and a VTI one represented by the nonhyperbolic formula of the first sequence of the Shanks transform given by a)

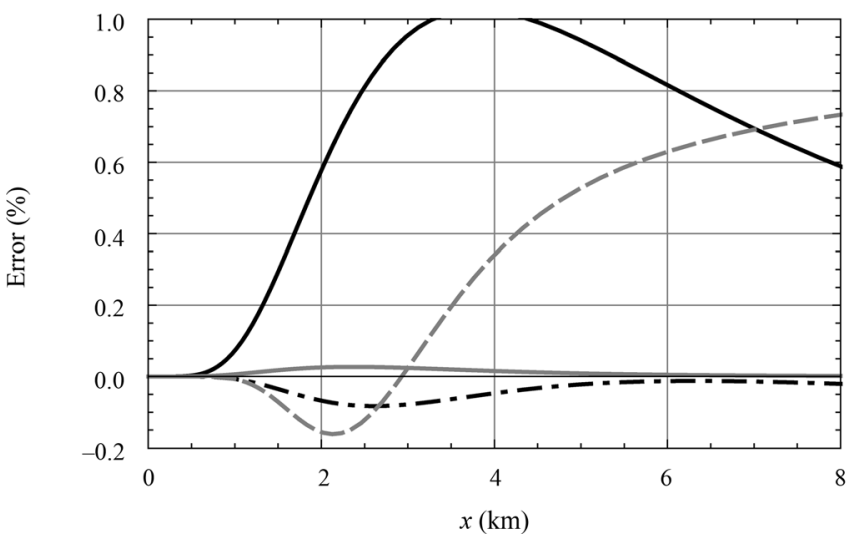

b)

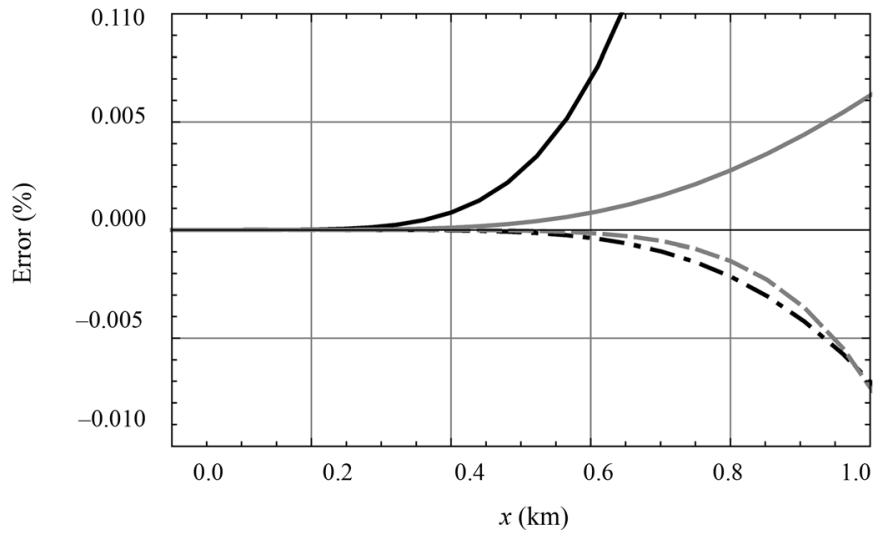

Figure 3. (a) The traveltime error as a function of offset for a model with $v=2 \mathrm{~km} / \mathrm{s}, \eta=0.2$, and a reflector depth of $z=2 \mathrm{~km}$ for the modified Hake representation (solid black curve), the shifted-hyperbola-based one (solid gray curve), the first sequence of the Shanks transform (dashed black curve), and the second sequence of the Shanks transform (dashed gray curve). (b) A closeup focusing on the moveout behavior near zero offset up to an offset-to-depth ratio of 0.5 . 
equation 8. Figure 5 shows the error in moveout when using a hyperbolic correction with the true NMO velocity in VTI media. It shows the influence of $\eta$ on moveout, which, as expected, increases with larger $\eta$ values and larger offsets. It also shows that the change in traveltime difference with a change in $\eta$, an important factor in the scan application, is more apparent for smaller $\eta$ values.

Although the homogeneous-medium versions of these equations show high accuracy, they are not meant to be used as an alternative to other available equations derived for the homogeneous case (i.e., Fomel's equation above). They were derived here only to test the potential accuracy of the newly developed formulas in their simplest representation, the homogeneous-medium case.

\section{THE ALGORITHM}

All of the traveltime perturbation equations developed above are linear first-order partial differential equations that can be solved using any of the many upwind numerical methods. Like Alkhalifah (2002), we rely on the fast marching method to solve such linear equations.

First we evaluate the background traveltime field using the eikonal equation or ray-based methods for a given source in an isotropic or elliptical anisotropic model. This traveltime field serves as the background field for predicting the traveltime for nonzero $\eta$ by solving a succession of linear first-order partial differential equations based on equation 5 , for the coefficients of the expansion in $\eta$.

To solve these first-order equations, we apply the fast marching numerical method to the equations derived from equation 5 , starting with $i=1$ to solve for $\tau_{1}$. We begin by evaluating the traveltime around the source for one layer of grid points for the case of first-order finite-difference approximation or two layers for the second-order case. This is accomplished using mainly point-to-point evaluations of traveltime. Next we start the fast marching using an update procedure based on an upwind firstor second-order approximation to the linear equations. In simple terms, the procedure starts with selecting one or more (as many as three) neighboring points around the updated point. The traveltime values at the selected neighboring points need to be

a)

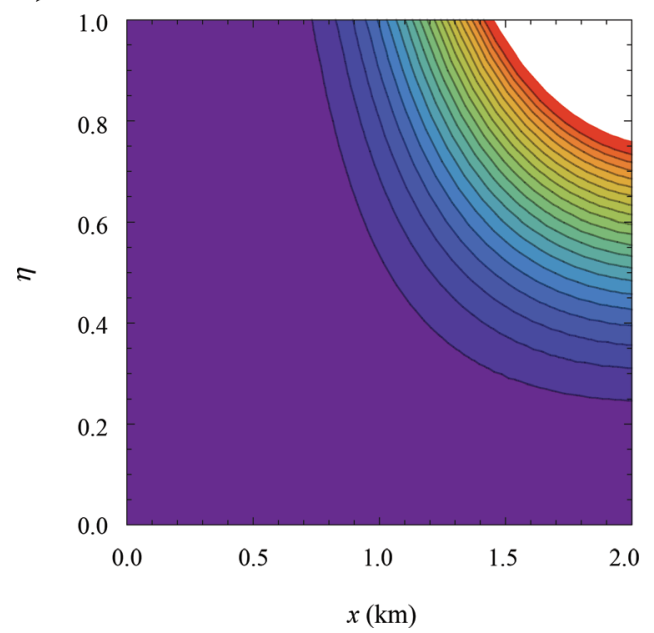

smaller than the current value. After the selection, one solves the discrete version of the linear partial differential equation for $\tau_{1}$. We add this perturbation traveltime value multiplied by $\eta$ to the background traveltime.

As the result of the updating process, a FarAway (newly evaluated traveltime) point is marked as NarrowBand or a NarrowBand (already on the front and includes a traveltime value) point gets assigned a new value. This process is repeated until we run out of points in the narrow band. A similar procedure is used for $\tau_{2}$ and then $\tau_{3}$ of equations 5 . The $\tau_{i}$ can serve to build the traveltime field for a known $\eta$ or to search for the best fit $\eta$ for a known traveltime field using equation A-2.

The solution of the first- and second-order fast marching, unlike the conventional eikonal, does not require evaluating a square root. This results from the linear nature of these equations and makes the evaluation faster.

\section{NUMERICAL TEST}

In this section, I test the accuracy of these linearized equations in computing traveltimes for VTI media starting with a background isotropic model. Thus, only perturbations in $\eta$ are examined, and the values of NMO velocity and vertical velocity are kept constant.

Consider a model that contains a lens anomaly in an otherwise constant-velocity-gradient $\left(d v / d x=0.5 \mathrm{~s}^{-1}\right.$ and $d v / d z$ $=0.7 \mathrm{~s}^{-1}$ with velocity at the origin equal to $2 \mathrm{~km} / \mathrm{s}$ ) model, with $v_{v}=v$ and $\eta=0.2$. The apex of the lens is located at a lateral position of 600 meters and depth of 500 meters with a velocity perturbation of $+500 \mathrm{~m} / \mathrm{s}$ (or 20\%) at the apex. The lens has a diameter of 200 meters, and as a result, the velocity variation needed to accommodate the perturbation is large. Using this model, we will test the accuracy of the first-order, second-order, and Shanks transform representation expansion equations.

For a source located at a lateral position of 200 meters from origin and a depth of 200 meters, we solve the isotropic eikonal equation using the fast marching method with second-order accuracy. The traveltime field in this case is represented by the

b)

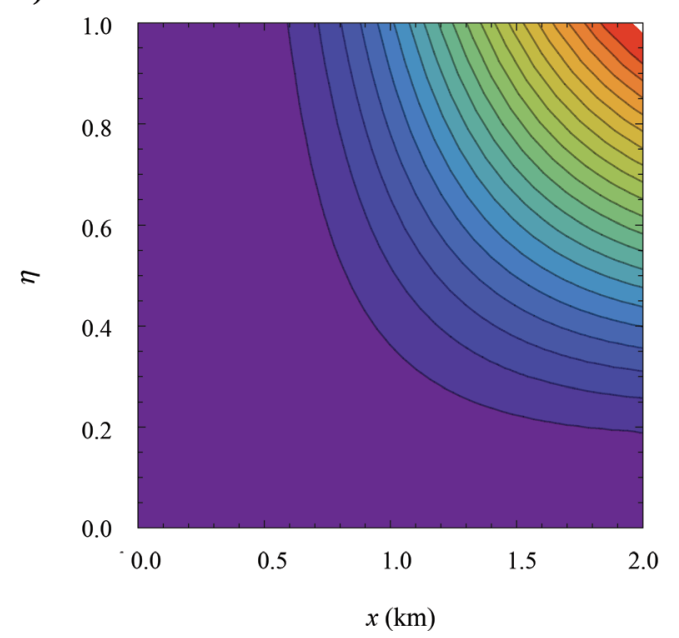

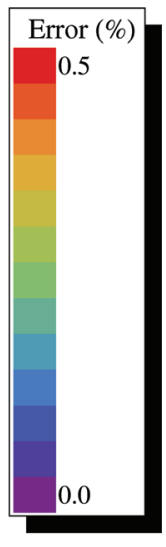

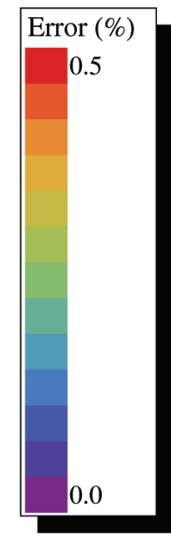

$x(\mathrm{~km})$

Figure 4. A color contour map of the traveltime errors for a model with $v=2 \mathrm{~km} / \mathrm{s}$ and a reflector of depth $z=2 \mathrm{~km}$ for (a) the second sequence of the Shanks transform and (b) the shifted-hyperbola-based formula. 


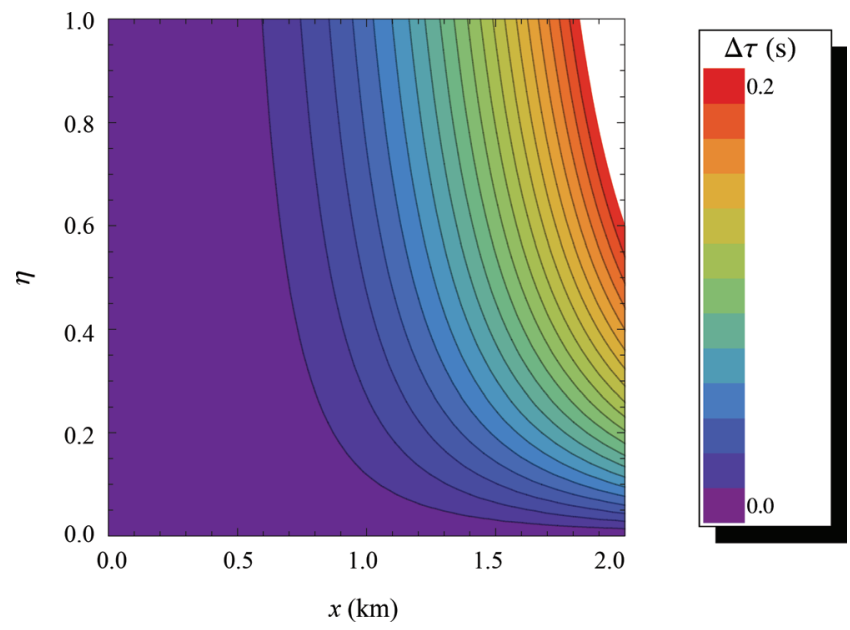

Figure 5. A color contour plot of the traveltime residual between a hyperbolic (isotropic) correction using the model NMO velocity of $2 \mathrm{~km} / \mathrm{s}$ and the moveout depicted by the nonhyperbolic expression of equation 8 . The reflector depth is again set to $2 \mathrm{~km}$. semisolid contours on the left-side plots of Figures 6,7 , and 8 . We also solve the anisotropic eikonal equation for $\eta=0.2$; it is represented by the semisolid contours in the middle plot of all three figures. Solving for $\tau_{1}$ using equation A-4 along with the original traveltime field, we obtain an approximate traveltime field for $\eta=0.2$. This new traveltime field is represented by the dashed contour curves in Figure 6. The absolute difference between the approximated traveltime and the true one (both displayed in the center plot) is given by the density plot shown on the right side of Figure 6. The errors are generally small (less than $0.008 \mathrm{~s}$ ), with the largest of these errors appearing around the vertical part of the wavefront. This error is generally small considering the large $\eta$ and first-order nature of the expansion.

Figure 7 is similar to Figure 6, but now we are using the second-order expansion obtained by solving equation A-5, which will require overall solving the linear partial differential equation twice. As expected, the errors are smaller than the first-order case with clear reduction in the near-vertical part of the wavefront.

With hardly any additional computational cost, we can use the Shanks transform representation of the expansion, and in this case the errors, as shown in Figure 8, are dramatically reduced, with errors apparent only in the shadow of the complex lens region.
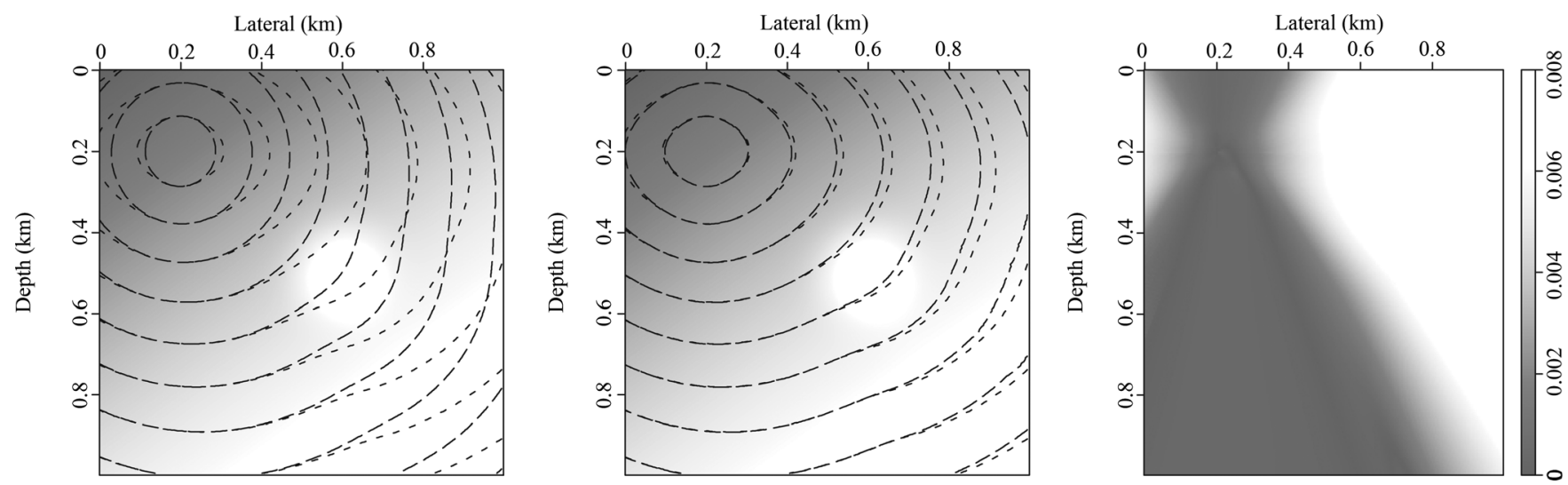

Figure 6. The traveltime contour (semisolid curve) plot for (left) an isotropic medium and (middle) an anisotropic medium, both compared with the traveltime derived using the first-order accuracy perturbation eikonal for $\eta=0.2$ (dashed curves). In both plots, the velocity field is shown in the background. Shown on the right is a density plot of the difference between the two contours in the middle plot.
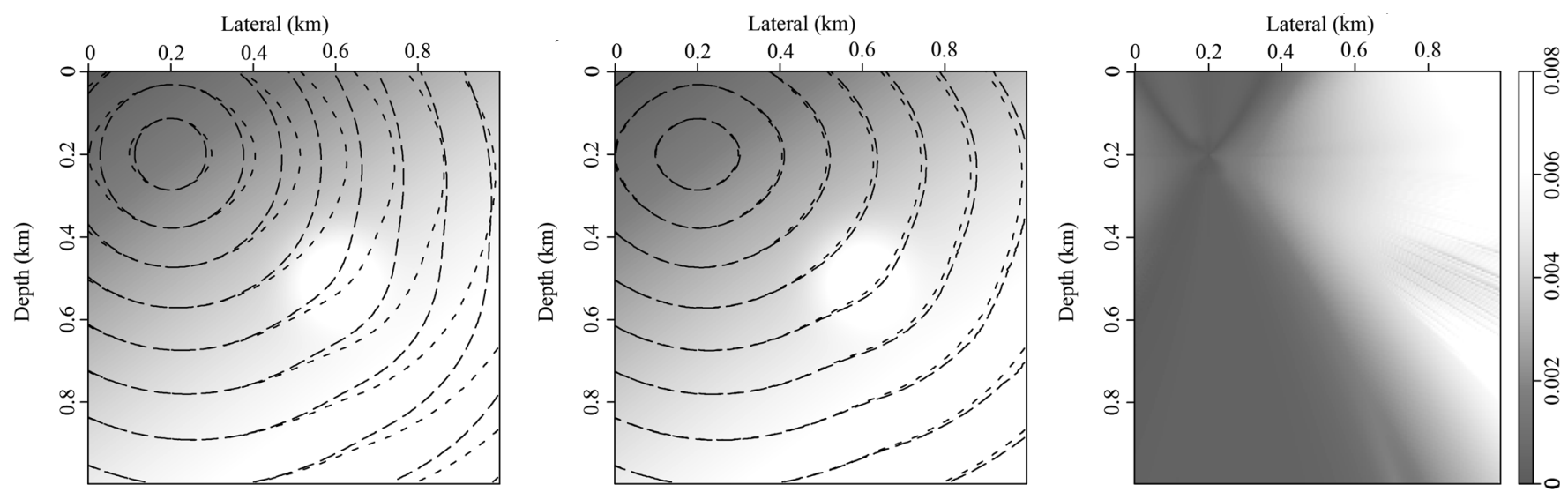

Figure 7. The traveltime contour (semisolid curve) plot for (left) an isotropic medium and (middle) an anisotropic medium, both compared with the traveltime derived using the second-order accuracy perturbation eikonal for $\eta=0.2$ (dashed curves). In both plots, the velocity field is shown in the background. Shown on the right is a density plot of the difference between the two contours in the middle plot. 
Clearly, from this experiment, we see the exceptional accuracy of this approach on a reasonably complicated model for a practical level of effective anisotropy. Of course, the accuracy of the Shanks transform representation is high, and, as a result, this representation should always be used. Next we apply the approach on a more complicated (Marmousi) model and introduce the concept of the $\eta$ scan.

\section{THE ANISOTROPIC SCAN}

Because we are expanding the traveltime solution in $\eta$, like many nonhyperbolic approximations for homogeneous media, we can evaluate the traveltime efficiently as a function of a constant $\eta$, but now for a generally inhomogeneous background medium. The set of linearized eikonal equations introduced here to predict traveltime for VTI media has the potential to help us estimate anisotropy parameters in complex media. Simply stated, by precomputing the coefficients of the $\eta$ expansion, we can directly assemble many traveltime solutions for a range of $\eta$, analytically. This also allows us to develop semblance-based displays with the horizontal axis representing $\eta$ instead of velocity.

In this case, we use the Marmousi model to test these assertions for the generally inhomogeneous case. For image-gather analysis (or exploding reflector assumption), we set a source at an image point in the Marmousi model. This source is located in the assumed exploration target area for this model given by a reservoir at a depth of 2400 meters. Figure 9 shows the traveltime contours from such a source for the isotropic case (elongated dashed curves) and for $\eta=0.1$ calculated using a finite-difference approximation of the VTI eikonal equation (solid curve) and the linear differential equations with the Shanks transform (dashed curves). The difference between the isotropic curves and the anisotropic curves is obvious for the vertical part of the wavefront corresponding to horizontal propagation. The linearized approximation provides reasonable accuracy for nonvertical wavefronts, and especially those approaching the surface.

The traveltime response on the surface for the source in Figure 9 is displayed in Figure 10 for a range of $\eta$ values, all computed using the Shanks transform analytic formula, equation 6 . The coefficients of the expansion are computed using equations 5 with the Marmousi velocity model. The range of $\eta$ used here between 0 and 0.5 has a reasonably large influence on the traveltime field with the fastest arrivals near the edges corresponding to $\eta=0.5$. Note that the smallest traveltime is not above the source at $6700 \mathrm{~m}$, which is a testament to the complexity of the velocity field. It is interesting also that the traveltime perturbation is apparent even for the horizontal part of the wavefront.

\section{DISCUSSION}

Kirchhoff prestack migration usually is applied using precomputed traveltime tables, computed often considering an isotropic inhomogeneous model. If the image gathers show misalignment of reflections as a function of offset, we will need to update the velocity model. In some cases, the update includes moving to an anisotropic model with the same degree of background velocity inhomogeneity. This requires perturbation in only $\eta$ and thus implies that we can use the above expansion to scan for such an $\eta$. As a result, instead of calculating the traveltime for an anisotropic model, we can perturb from the isotropic (or elliptical anisotropic) velocity model to include VTI anisotropy. For anisotropic media, using the linearization process reduces the cost associated with traveltime calculation in anisotropic media. In velocity estimation, the cost saving is magnified by the fact that we will need to calculate traveltimes in anisotropic media for different, yet close, velocity models. Traveltime perturbation is a shortcut that provides us with traveltimes for these various models using an analytic formula.

To scan for $\eta$ in a full 3D data set requires computing three traveltime fields for each (or targeted) sources and receivers, which will require a large traveltime table. However, because this is used to scan for $\eta$, the coarse sampling of sources and targeting certain regions can reduce the amount of data required. Another feature of the above formulation is that the background traveltime field for the elliptically (or isotropic) model can come from any algorithm. For example, we can use ray tracing or any multivalued eikonal to obtain multiarrival fields and perturb those to maintain the branches.

Developing the background velocity model for the isotropic or elliptical anisotropic case can be done using many commonly known methods. In addition to approximating the tilt by the
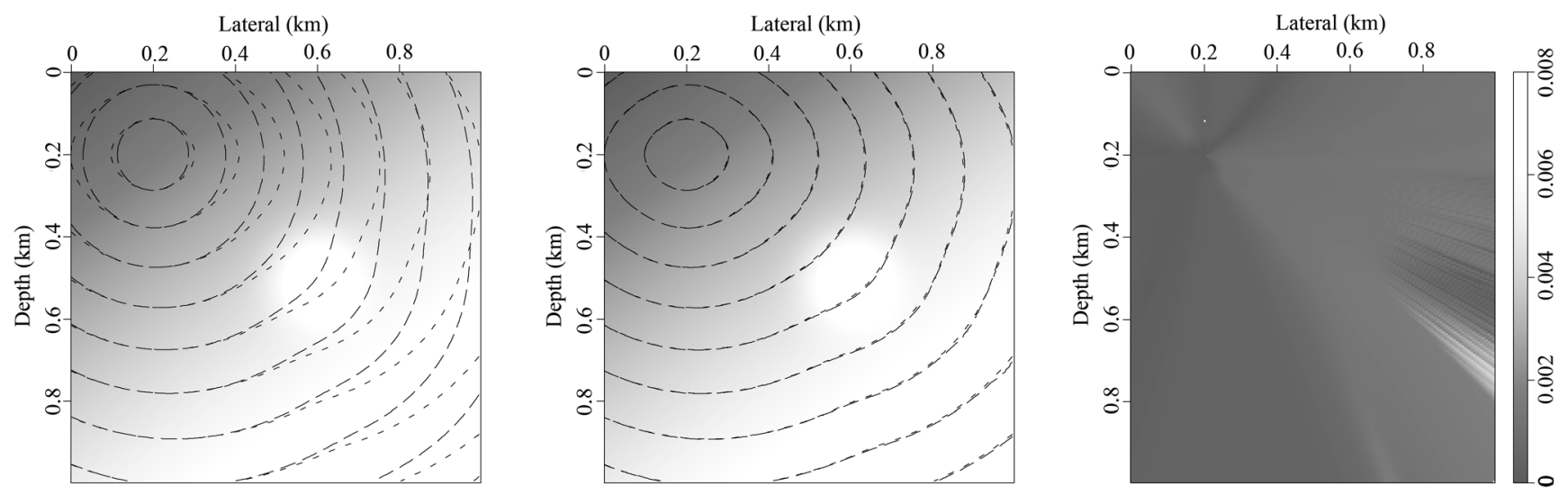

Figure 8. The traveltime contour (semisolid curve) plot for (left) an isotropic medium and (middle) an anisotropic medium, both compared with the traveltime derived using the Shanks transform perturbation eikonal for $\eta=0.2$ (dashed curves). In both plots, the velocity field is shown in the background. Shown on the right is a density plot of the difference between the two contours in the middle plot. 
structure of the model, velocities are evaluated using stacking velocity analysis and check-shot well log measurements or vertical seismic profiling (VSP).

For TI media with a tilt in the axis of symmetry, two additional parameters $\theta$ and $\phi$ that describe the tilt in three dimensions are needed to fully characterize a high-frequency acoustic wave propagation. These two parameters often are estimated by assuming that the tilt direction is normal to the medium structure or in the direction of the velocity gradient (Alkhalifah and Bednar, 2000). This leaves us with a similar number of unknown parameters as in the VTI case. However, our axis-ofsymmetry vertical velocity is no longer vertical, and thus, it will have more influence on data recorded at the surface.

For the traveltime equations in Appendix A to work for a tilted axis of symmetry, a simple coordinate transformation to the traveltime derivatives is applied based on the following Jacobian in three dimensions:

$$
\left(\begin{array}{ccc}
\cos (\phi) \cos (\theta) & \sin (\phi) \cos (\theta) & \sin (\theta) \\
-\sin (\phi) & \cos (\phi) & 0 \\
-\cos (\phi) \sin (\theta) & \sin (\phi) \sin (\theta) & \cos (\theta)
\end{array}\right)
$$

where $\theta$ is measured from the vertical and $\phi$ from the $x$-axis. Setting $\phi=0$ yields the form for the 2D case. Because this

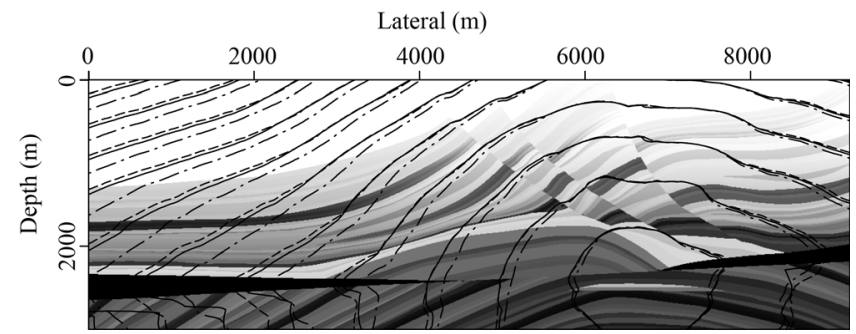

Figure 9. Traveltime contours for the isotropic Marmousi model (elongated dashed curves) and for $\eta=0.1$ using an anisotropic VTI eikonal solver (solid curves) and the Shanks transform expansion (dashed curves) for a source located at the lateral position of $6700 \mathrm{~m}$ and depth $2400 \mathrm{~m}$. The velocity Marmousi model is shown in the background.

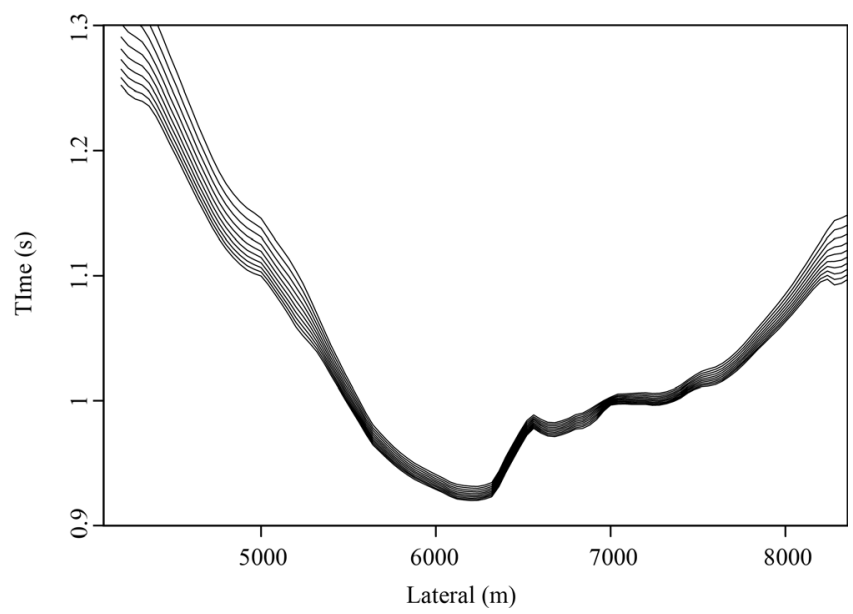

Figure 10. Traveltime as a function of lateral position measured at the surface (depth equals 0) for the source shown in Figure 9 for a range of $\eta$ between zero and 0.5 at an increment of 0.05 . coordinate transform is linear and the perturbation differential equations are linear as well, then the resulting equations for TTI media are linear. In this case also, the background medium is an elliptically anisotropic model with a tilted axis of symmetry, which is easily solvable using common numerical techniques. The challenge here, however, is parameter estimation in TTI and in particular getting the NMO velocity and the tilt-direction velocity for the elliptical background model.

\section{CONCLUSIONS}

Expanding the traveltime solutions of the VTI eikonal equation in a power series in terms of the independent anisotropic parameter $\eta$ provides an efficient tool to estimate $\eta$ in a generally inhomogeneous background medium. The Shanks transform allows for better representation using fewer terms of the expansion, and thus fewer equations to solve. The simplified representation for a homogeneous background results in analytic nonhyperbolic moveout equations for anisotropic media; the representation is exceptional in its simplicity and accuracy. Yet, the formulation provides $\eta$ estimation capability for a generally inhomogeneous background medium. Testing the general form on the Marmousi model proved its versatility and general accuracy. The TTI version requires a simple coordinate transformation and allows for $\eta$ scans after developing a tilted elliptical anisotropic model.

\section{ACKNOWLEDGMENTS}

I am grateful to King Abdullah University of Science and Technology (KAUST) and KACST for its financial support, and I thank Alexey Stovas, Paul Sava, Samuel Gray, and four anonymous reviewers for their critical and helpful review of the paper.

\section{APPENDIX A \\ HIGHER ORDER EXPANSIONS}

To derive a traveltime equation in terms of perturbations in $\eta$, we first establish the form for the governing equation for VTI media given by the eikonal representation. The eikonal equation, as derived by Alkhalifah (1998), is given by

$$
\begin{gathered}
(1+2 \eta) v^{2}\left(\left(\frac{\partial \tau}{\partial x}\right)^{2}+\left(\frac{\partial \tau}{\partial y}\right)^{2}\right)+v_{v}^{2}\left(\frac{\partial \tau}{\partial z}\right)^{2} \\
-2 \eta v^{2} v_{v}^{2}\left(\left(\frac{\partial \tau}{\partial x}\right)^{2}+\left(\frac{\partial \tau}{\partial y}\right)^{2}\right)\left(\frac{\partial \tau}{\partial z}\right)^{2}=1 .
\end{gathered}
$$

To solve equation A-1 through perturbation theory, we assume that $\eta$ is small, and thus a trial solution can be expressed as a series expansion in $\eta$ :

$$
\begin{aligned}
\tau(x, y, z) \approx & \tau_{0}(x, y, z)+\tau_{1}(x, y, z) \eta+\tau_{2}(x, y, z) \eta^{2} \\
& +\tau_{3}(x, y, z) \eta^{3}
\end{aligned}
$$

where $\tau_{0}, \tau_{1}, \tau_{2}$, and $\tau_{3}$ are coefficients of the expansion given in units of traveltime, and, for practicality, terminated at the cubic power of $\eta$. Inserting the trial solution, equation A-2, into equation A-1 yields a long formula, but by setting $\eta=0$, we obtain the 
zeroth-order term given by

$$
v^{2}(x, y, z)\left(\left(\frac{\partial \tau_{0}}{\partial x}\right)^{2}+\left(\frac{\partial \tau_{0}}{\partial y}\right)^{2}\right)+v_{v}^{2}(x, y, z)\left(\frac{\partial \tau_{0}}{\partial z}\right)^{2}=1,
$$

which is the eikonal formula for elliptical anisotropy. By equating the coefficients of the powers of the independent parameter $\eta$, in succession, we end up first with the coefficients of first power in $\eta$, simplified by using equation A-3, given by

$v^{2} \frac{\partial \tau_{0}}{\partial x} \frac{\partial \tau_{1}}{\partial x}+v^{2} \frac{\partial \tau_{0}}{\partial y} \frac{\partial \tau_{1}}{\partial y}+v_{v}^{2} \frac{\partial \tau_{0}}{\partial z} \frac{\partial \tau_{1}}{\partial z}=-\left(1-v_{v}^{2}\left(\frac{\partial \tau_{0}}{\partial z}\right)^{2}\right)^{2}$

which is a first-order linear partial differential equation in $\tau_{1}$. The coefficients of the square terms in $\eta$, with some manipulation, result in the following relation:

$$
\begin{aligned}
v^{2} & \frac{\partial \tau_{0}}{\partial x} \frac{\partial \tau_{2}}{\partial x}+v^{2} \frac{\partial \tau_{0}}{\partial y} \frac{\partial \tau_{2}}{\partial y}+v_{v}^{2} \frac{\partial \tau_{0}}{\partial z} \frac{\partial \tau_{2}}{\partial z} \\
= & -\frac{1}{2}\left(v^{2}\left(\frac{\partial \tau_{1}}{\partial x}\right)^{2}+v^{2}\left(\frac{\partial \tau_{1}}{\partial y}\right)^{2}+v_{v}^{2}\left(\frac{\partial \tau_{1}}{\partial z}\right)^{2}\right) \\
& +2\left(v_{v}^{2} \frac{\partial \tau_{0}}{\partial z} \frac{\partial \tau_{1}}{\partial z}-v^{2}\left(\frac{\partial \tau_{0}}{\partial x} \frac{\partial \tau_{1}}{\partial x}+\frac{\partial \tau_{0}}{\partial y} \frac{\partial \tau_{1}}{\partial y}\right)\right) \\
& \times\left(1-v_{v}^{2}\left(\frac{\partial \tau_{0}}{\partial z}\right)^{2}\right)
\end{aligned}
$$

which is again a first-order linear partial differential equation in $\tau_{2}$ with an obviously more complicated source function given by the right-hand side.

Finally, the coefficients of the cubic terms in $\eta$ result in a firstorder linear partial differential equation in $\tau_{3}$ given by

$$
\begin{aligned}
v^{2} \frac{\partial \tau_{0}}{\partial x} \frac{\partial \tau_{3}}{\partial x} & +v^{2} \frac{\partial \tau_{0}}{\partial y} \frac{\partial \tau_{3}}{\partial y}+v_{v}^{2} \frac{\partial \tau_{0}}{\partial z} \frac{\partial \tau_{3}}{\partial z} \\
= & 4 v_{v}^{2} v^{2} \frac{\partial \tau_{0}}{\partial z} \frac{\partial \tau_{1}}{\partial z}\left(\frac{\partial \tau_{0}}{\partial x} \frac{\partial \tau_{1}}{\partial x}+\frac{\partial \tau_{0}}{\partial y} \frac{\partial \tau_{1}}{\partial y}\right) \\
& +2\left(v_{v}^{2} \frac{\partial \tau_{0}}{\partial z} \frac{\partial \tau_{2}}{\partial z}-v^{2}\left(\frac{\partial \tau_{0}}{\partial x} \frac{\partial \tau_{2}}{\partial x}+\frac{\partial \tau_{0}}{\partial y} \frac{\partial \tau_{2}}{\partial y}\right)\right) \\
& \times\left(1-v_{v}^{2}\left(\frac{\partial \tau_{0}}{\partial z}\right)^{2}\right) \\
& +\left(v_{v}^{2}\left(\frac{\partial \tau_{1}}{\partial z}\right)^{2}-v^{2}\left(\frac{\partial \tau_{1}}{\partial x}\right)^{2}-v^{2}\left(\frac{\partial \tau_{1}}{\partial y}\right)^{2}\right) \\
& \times\left(1-v_{v}^{2}\left(\frac{\partial \tau_{0}}{\partial z}\right)^{2}\right) \\
& -v^{2} \frac{\partial \tau_{1}}{\partial x} \frac{\partial \tau_{2}}{\partial x}-v^{2} \frac{\partial \tau_{1}}{\partial y} \frac{\partial \tau_{2}}{\partial y}-v_{v}^{2} \frac{\partial \tau_{1}}{\partial z} \frac{\partial \tau_{2}}{\partial z}
\end{aligned}
$$

Although the equation seems complicated, many of the variables of the source function (right-hand side) can be evaluated during the evaluation of equations A-4 and A-5 in a fashion that will not add much to the cost.
Using the Shanks transforms (Bender and Orszag, 1978), we can isolate and remove the most transient behavior of the expansion A-2 by first defining the following parameters:

$$
\begin{aligned}
& A_{0}=\tau_{0}, \\
& A_{1}=\tau_{0}+\tau_{1} \eta, \\
& A_{2}=\tau_{0}+\tau_{1} \eta+\tau_{2} \eta^{2}, \\
& A_{3}=\tau_{0}+\tau_{1} \eta+\tau_{2} \eta^{2}+\tau_{3} \eta^{3} .
\end{aligned}
$$

The first sequence of the Shanks transforms uses only $A_{0}, A_{1}$, and $A_{2}$, and thus is given by

$$
\begin{aligned}
\tau(x, y, z) & \approx \frac{A_{0} A_{2}-A_{1}^{2}}{A_{0}-2 A_{1}+A_{2}} \\
& =\tau_{0}(x, y, z)+\frac{\eta \tau_{1}^{2}(x, y, z)}{\tau_{1}(x, y, z)-\eta \tau_{2}(x, y, z)} .
\end{aligned}
$$

On the other hand, the second sequence (higher order) Shanks transform uses $A_{1}, A_{2}$, and $A_{3}$, and thus is given by

$$
\begin{aligned}
\tau(x, y, z) \approx & \frac{A_{1} A_{3}-A_{2}^{2}}{A_{1}-2 A_{2}+A_{3}}=\tau_{0}(x, y, z) \\
& +\eta\left(\tau_{1}(x, y, z)+\frac{\eta \tau_{2}^{2}(x, y, z)}{\tau_{2}(x, y, z)-\eta \tau_{3}(x, y, z)}\right) .
\end{aligned}
$$

\section{APPENDIX B}

\section{THE HOMOGENEOUS-MEDIUM CASE}

To develop the traveltime relation for VTI media, we start with a background velocity model that is homogeneous. At this stage, we will consider $v_{v}=v$ to simplify the derivation. In this case, the traveltime is given by the following simple relation in two dimensions:

$$
\tau_{0}(x, z)=\frac{\sqrt{x^{2}+z^{2}}}{v}
$$

which satisfies the eikonal equation A-3. Using equation B-1, we evaluate $\partial \tau_{0} / \partial x$ and $\partial \tau_{0} / \partial z$ and insert them into equation A-4 to solve the first-order linear equation to obtain

$$
\tau_{1}(x, z)=-\frac{x^{4}}{v\left(x^{2}+z^{2}\right)^{3 / 2}} .
$$

We now evaluate $\partial \tau_{1} / \partial x$ and $\partial \tau_{1} / \partial z$ and use them to solve equation A-5. After some tedious algebra, we obtain

$$
\tau_{2}(x, z)=\frac{3\left(x^{8}+4 x^{6} z^{2}\right)}{2 v\left(x^{2}+z^{2}\right)^{7 / 2}}
$$

Finally, we evaluate $\partial \tau_{2} / \partial x$ and $\partial \tau_{2} / \partial z$ and use them to solve equation

A-6. After some tedious algebra again, we obtain

$$
\tau_{3}(x, z)=-\frac{x^{8}\left(5 x^{4}+28 x^{2} z^{2}+104 z^{4}\right)}{2 v\left(x^{2}+z^{2}\right)^{11 / 2}} .
$$


Using the first sequence of the Shanks transform, equation A-8, applied to the Taylor series expansion, we obtain the following:

$$
\tau(x, z)=\frac{\sqrt{x^{2}+z^{2}}\left((\eta+2) x^{4}+4(3 \eta+1) x^{2} z^{2}+2 z^{4}\right)}{v\left((3 \eta+2) x^{4}+4(3 \eta+1) x^{2} z^{2}+2 z^{4}\right)} .
$$

The second sequence of the Shanks transform results in

$$
\begin{aligned}
\tau(x, z)=\frac{\sqrt{x^{2}+z^{2}}}{2 v\left(x^{2}+z^{2}\right)^{2}} & \\
& \times\left(\frac{(6-(\eta-4) \eta) x^{10}+8(\eta(2 \eta+5)+6) x^{8} z^{2}+4((69-16 \eta) \eta+33) x^{6} z^{4}}{\left((5 \eta+3) x^{6}+2(14 \eta+9) x^{4} z^{2}+(104 \eta+27) x^{2} z^{4}+12 z^{6}\right)}\right. \\
& \left.+\frac{56(8 \eta+3) x^{4} z^{6}+2(104 \eta+51) x^{2} z^{8}+24 z^{10}}{\left((5 \eta+3) x^{6}+2(14 \eta+9) x^{4} z^{2}+(104 \eta+27) x^{2} z^{4}+12 z^{6}\right)}\right) . \quad \text { (B-6) }
\end{aligned}
$$

For 3D media, we replace $x$ with $r=\sqrt{x^{2}+y^{2}}$.

\section{REFERENCES}

Aldridge, D. F., 1994, Linearization of the eikonal equation: Geophysics, 59, 1631-1632, doi:10.1190/1.1443552.

Alkhalifah, T., 1997, Velocity analysis using nonhyperbolic moveout in transversely isotropic media: Geophysics, 62, 1839-1854, doi:10.1190/ 1.1444285 .

1998, Acoustic approximations for processing in transversely isotropic media: Geophysics, 63, 623-631, doi:10.1190/1.1444361.

-2000, An acoustic wave equation for anisotropic media: Geophysics, 65, 1239-1250, doi:10.1190/1.1444815.

, 2002, Traveltime computation with the linearized eikonal equation for anisotropic media: Geophysical Prospecting, 50, 373-382, doi: 10.1046/j.1365-2478.2002.00322.x.
Alkhalifah, T., and J. Bednar, 2000, Building a 3-D anisotropic model: Its implications to traveltime calculation and velocity analysis: 70th Annual International Meeting, SEG, Expanded Abstracts, 965-968.

Alkhalifah, T., and S. Fomel, 2001, Implementing the fast marching eikonal solver: Spherical versus Cartesian coordinates: Geophysical Prospecting, 49, 165-178, doi:10.1046/j.1365-2478.2001.00245.x.

Alkhalifah, T., and I. Tsvankin, 1995, Velocity analysis for transversely isotropic media: Geophysics, 60, 1550-1566, doi:10.1190/1.1443888.

Al-Yahya, K. M., 1989, Velocity analysis by iterative profile migration: Geophysics, 54, 718-729, doi:10.1190/1.1442699.

Audebert, F., J. P. Diet, P. Guillaume, I. F. Jones, and X. Zhang, 1997, CRpscans: 3-D preSdM velocity analysis via zero-offset tomographic inversion: 67th Annual International Meeting, SEG, Expanded Abstracts, 1805-1808.

Bender, C. M., and S. A. Orszag, 1978, Advanced mathematical methods for scientists and engineers: McGraw-Hill.

Dellinger, J., and F. Muir, 1988, Imaging reflections in elliptically anisotropic media: Geophysics, 53, 1616-1618, doi:10.1190/1.1442446.

Ettrich, N., and D. Gajewski, 1998, Traveltime computation by perturbation with FD-eikonal solvers in isotropic and weakly anisotropic media: Geophysics, 63, 1066-1078, doi:10.1190/1.1444385.

Fomel, S., 2004, On anelliptic approximations for qP velocities in VTI media: Geophysical Prospecting, 52, 247-259, doi:10.1111/ j.13652478.2004.00413.x.

Hake, H., K. Helbig, and C. S. Mesdag, 1984, Three-term Taylor series for $t^{2}-x^{2}$-curves of P-waves and S-waves over layered transversely isotropic ground: Geophysical Prospecting, 32, 828-850, doi:10.1111/ j.13652478.1984.tb00742.x

Peng, C., and K. Steenson, 2001, 3-D prestack depth migration in anisotropic media: A case study at the Lodgepole reef play in North Dakota: The Leading Edge, 20, 524-527, doi:10.1190/1.1438986.

Popovici, M., 1991, Finite difference travel time maps: Stanford Exploration Project Report 70, 245-256.

Sethian, J. A., 1996, A fast marching level set method for monotonically advancing fronts: Proceedings of the National Academy of Sciences of the United States of America, 93, no. 4, 1591-1595, doi:10.1073/pnas. 93.4.1591.

van Trier, J., and W. W. Symes, 1991, Upwind finite-difference calculation of traveltimes: Geophysics, 56, 812-821, doi:10.1190/ 1.1443099 .

Vidale, J. E., 1990, Finite-difference calculation of traveltimes in three dimensions: Geophysics, 55, 521-526, doi:10.1190/1.1442863. 